\title{
Perspectives on the Management of Abdominal Trauma
}

\author{
Weledji EP ${ }^{1 *}$ and Tambe $J^{2}$ \\ ${ }^{1}$ Department of Surgery, University of Buea, Cameroon \\ 2Imaging Centre, Regional Hospital, Cameroon \\ *Corresponding author: Elory Patrick Weledji, \\ Department of Surgery, Faculty of Health Sciences, \\ University of Buea, Cameroon
}

Received: July 09, 2018; Accepted: July 31, 2018; Published: August 07, 2018

\begin{abstract}
The main consequences of abdominal trauma are haemorrhage and sepsis. Early deaths following abdominal trauma are usually attributable to haemorrhage. Sepsis is the most common cause in deaths occurring more than 48 hours after injury. Thus the first priority for the surgeon performing a laparotomy for abdominal trauma is haemorrhage control and prevention of spilling of visceral contents from visceral injuries is the second priority. In selected patients definitive repair is delayed until after a period of intensive resuscitation following damage-control surgery. The diagnosis or exclusion of hollow viscus injuries can be problematic. Excluding the general principles of trauma laparotomy and definitive intra-abdominal procedures, the article discussed the clinical assessment and decision-making, which would ensure that injuries are not missed during laparotomy and thus decrease mortality.
\end{abstract}

Keywords: Abdomen; Trauma; Assessment; Adjuncts; Laparotomy; Damage-control

\section{Introduction}

In civilian practice approximately $20 \%$ of trauma injuries requiring surgery involve the abdomen [1]. Abdominal trauma may be blunt or penetrating, but generally, in civilian practice, blunt trauma is more common than penetrating and usually follows a road traffic crash. However, in the American urban civilian practice penetrating trauma is more common than blunt trauma, gunshot wounds being more frequent than stab wounds [2]. In the UK stab wounds predominate [3]. In military practice, penetrating abdominal wounds are greater than blunt with a high mortality from the high velocity missile/ bullet/ fragment wounds [4]. The diagnosis of abdominal injury by clinical examination is unreliable and, thus in the initial management of abdominal trauma in adults following rapid assessment and resuscitation selection of appropriate investigations is of key importance $[5,6]$.

\section{Discussion}

\section{Patterns of injuries}

Particular pattern of injuries occur with blunt abdominal trauma Steering wheel injuries commonly involve the sternum (with the risk of myocardial contusion), liver and spleen. Pelvic fractures are associated with urethral and urinary bladder injuries and with rupture of the diaphragm. Different types of lumbar vertebral fractures from acceleration/ deceleration injuries are associated with various abdominal injuries. Transverse spinous process fractures may occur with renal trauma and horizontal fractures of the vertebrae through the body are associated with pancreatic, duodenal or small bowel mesentery injuries. It is important to understand the concept of the trimodal distribution of death (\%) during a road traffic crash [1]. The first phase is death within seconds to minutes (40\%) from the impact of the crash (energy being converted from one form to the other according to the first law of thermodynamics) causing instant damage to the brain, heart and great vessels and cervical cord. The second phase is the 'golden hour' as death occurs within minutes to hours $(30 \%)$ and thus can clinically be acted upon, influenced and death prevented. This 'golden hour' phase forms the basis of the primary survey (ABCDE) of the Advanced Trauma Life Support (ATLS) system of management in which immediately lifethreatening injuries are identified and treated in the correct order [79]. It consists of A- airway with cervical spine control, B-breathing with oxygen, C- circulation with haemorrhage control, D- disability of the central nervous system, E-exposure looking for hidden injury or signs of external bleeding but taking care to avoid hypothermia). The injury in this phase may involve the brain (treatable haematoma), lungs (haemo and pneumothorax), abdomen (haemorrhage) and skeleton (pelvis and long bones). The third phase is death within days to weeks (30\%) from infection, multiple organ failure, abdomen (haemorrhage) and injury to the skeleton (pelvis and long bones).

\section{Pathophysiology}

The main consequences of abdominal trauma are haemorrhage and sepsis. Early deaths following abdominal trauma are usually attributable to haemorrhage. Blood can be surprisingly non-irritant and large volumes can be sequestered within the abdominal cavity before any clinical signs become evident. Blunt trauma frequently causes injuries to solid organs such as the spleen and liver. The energy transfer during deceleration and compression tears solid organs and bowel mesentery resulting in intraperitoneal bleeding. Shearing forces caused by deceleration lead to organs and tissues moving relative to each other at the interface between mobile and fixed structures such as the proximal intra-peritoneal and distal retroperitoneal first part of the duodenum and blood vessels tearing at these junctions. Penetrating trauma from stabbing, bullets and fragments causes haemorrhage from solid organs as well as the major arteries and veins in the abdomen. Sepsis is the most common cause of death occurring more than 48 hours after injury. Hollow viscus injury with leakage of gut contents is the usual cause of intra-abdominal sepsis after trauma and occurs frequently with penetrating trauma. In stab wounds it is easier to predict the injured organs as the injuries are usually confined to the tract [3]. However, with gunshot wounds it is never safe to assume that the missile has followed a straight trajectory. In addition, 
high velocity missile wounds have a cavitation effect that may extend several centimetres beyond the tract of the missile. Blunt abdominal trauma can also result in rupture of intra-abdominal, retroperitoneal and pelvic hollow viscera. Blast is a potent cause of blunt trauma in military practice, especially affecting gas-filled viscera $[4,9]$. Retroperitoneal injuries (pancreatic, duodenal, ureteric, ascending and descending colon) are often diagnosed late as these injuries can take days to become clinically apparent [10-15]. Thus, a patient who has been stabbed in the back should be watched for at least 48 hours to exclude signs of sepsis. Bladder injuries can have a delayed diagnosis as urine is non-irritant to the peritoneum. When the diagnosis of a ruptured bladder is missed, the patient may have been drunk or reluctant to seek medical attention, perhaps for social reasons.The diagnosis is considered if there is a rise in urea and creatinine levels, an absence of normal voiding and ill- defined abdominal tenderness [11-14]. Rectal injuries from penetrating trauma or associated pelvic fractures can be similarly missed, with resultant pelvic and systemic sepsis [15-17].

\section{Assessment}

The initial assessment and resuscitation of the injured patient should follow the ATLS sequence of airway, breathing and circulation as airway compromise causes death within seconds, breathing derangement causes death within minutes and circulatory impairment causes death within hours $[7,8]$. Shock, in the presence of obvious abdominal injuries, should prompt a laparotomy for haemorrhage control (resuscitation laparotomy) during the circulation stage of the primary survey. The assessment of the trauma patient following resuscitation includes obtaining a detailed history of the event from pre-hospital personnel. Knowledge of accident details (e.g. use of seat belts, estimated speeds, injuries to other passengers or any deaths) may enable the clinician to build a picture of likely injury patterns [7$9,18]$. A thorough examination of the abdomen is part of the secondary survey and must include rectal, penile and vaginal examination [7]. Physical examination of the abdomen in the trauma patient is unreliable and a single negative examination does not exclude serious injury. Regular review and documentation of findings are therefore essential as physical findings may undergo subtle changes with time $[6-8,19]$. Many injuries are not an immediate threat to life but will become fatal if not diagnosed and treated expeditiously. Thus, the role of the secondary survey [7]. The decision on which injuries mandate an urgent operation apart from obvious and exsanguinating bleeding is frequently difficult and best made by an experienced surgeon [1$3,19]$.

\section{Adjuncts to clinical examination}

Unlike penetrating abdominal trauma, where management is largely determined clinically, the diagnosis of blunt abdominal injury by clinical examination is unreliable, particularly in patients with a decreased level of consciousness [5,20-22]. Confirmation of the presence or absence of injury therefore relies largely on the use of diagnostic adjuncts. Accurate imaging facilitates selection for non-operative management, where appropriate, and reduces nontherapeutic laparotomy rates. The main first line investigations are ultrasound, diagnostic peritoneal lavage, and computed tomography. These tests are complementary rather than interchangeable, and their usefulness depends on the clinical context. Diagnostic Peritoneal Lavage (DPL) first described in 1965 [23]. Rapidly became the standard of care until the advent of Focused Assessment with Sonography for Trauma (FAST) in the resuscitation room. DPL detects blood in the peritoneal cavity with a sensitivity of $90 \%$ and an accuracy of $97 \%$ [23]. It is not as blind a procedure as the obsolete and dangerous four-quadrant tap. DPL is performed using either an open or a closed percutaneous technique through the umbilicus as a bedside manoeuvre under local anaesthetic. A lavage cell count of 100,000 red cells per $\mathrm{mm}^{3}$ indicates a clinically relevant haemoperitoneum whereas the presence of $>500$ white blood cells/ $\mathrm{mm}^{3}$ or vegetable matter signifies a hollow viscus injury [24]. The presence of any of these parameters is regarded as an indication for laparotomy $[25,26]$. Although, the technique has been shown to be reliable, reproducible, it does not exclude retroperitoneal injury and it is not performed in children. In addition, the infusion of lavage fluid, which is never completely removed, may also interfere with the interpretation of subsequent imaging. Since not all patients with a haemoperitoneum need laparotomy, the biggest drawback of diagnostic peritoneal lavage is the resulting high non-therapeutic laparotomy rate of up to $36 \%$ [26]. However, DPL may continue to have a role as a second line investigation in the neurologically compromised patient to clarify the nature of the fluid and in the diagnosis of hollow viscus injuries [25]. The sole purpose of FAST is to detect free fluid (which is mostly assumed to be blood and less to gastrointestinal content) in the peritoneal cavity after blunt trauma [27-29]. The sensitivity of FAST in abdominal trauma is $88 \%$ and it is therefore an ideal screening investigation for all trauma patients who do not need to go directly to theatre and patients who are unstable because of its rapid assessment [28]. A normal FAST does not exclude injury as signs of blood loss and hollow viscus injury may initially be subtle.If the patient remains cardio vascular stable, this can be augmented by Computed Tomography (CT) scan to either confirm the negative FAST or determine organ injury for non-operative management [27]. As it will also miss injuries not associated with intra-abdominal fluid, FAST may not be very useful in haemo dynamically stable patients $[28,29]$. It is therefore the investigation of choice in the haemodynamically unstable patient whereas CT is the investigation of choice in the haemo dynamically stable patient $[6,28,29]$. Patients who transiently respond to resuscitation should be managed as unstable patients. The decision to obtain a CT scan in such patients should be made only by experienced staff, after careful appraisal of the risks and potential benefits, and only if the results are likely to alter management.In unstable multiply injured patients, a positive FAST requires a laparotomy and a negative exam should either be repeated or a DPL performed. An unstable patient following a stab wound needs an immediate laparotomy but in the stable patient FAST should be used to detect free intraperitoneal bleeding. If this is negative, laparoscopy can be employed in the stable patient in the operating theatre. In the vast majority of patients laparoscopy has no role in the evaluation of abdominal trauma especially as it is time consuming and expensive [7]. It is also limited by technical factors such as the presence of blood and difficulty in fully assessing the retroperitoneal structures and small bowel. However, an indication for laparoscopy in blunt trauma is the assessment for diaphragm rupture in selected stable patients where there is clinical suspicion [7]. Rigid sigmoidoscopy is necessary if there is any suspicion of a rectal injury [16]. During the initial assessment, a nasogastric or orogastric tube should be inserted to decompress the stomach and 
reduce the probability of aspiration. A urinary catheter should also be inserted after rectal and perineal examination to avoid creation of a 'false' passage.Although some authorities continue to advocate its use $[24,30]$ abdominal radiography has no role in the assessment of blunt abdominal trauma. It may provide indirect evidence of hollow viscus injury by showing air or gas in the peritoneum, but lacks sensitivity and specificity.Chest and pelvic radiography continue to be important adjuncts to the primary survey. They may suggest haemorrhage in adjacent cavities, but they cannot rule out intra-abdominal bleeding or visceral injury [6].

\section{Decision making}

At this point in the assessment the management choices are: a) resuscitation laparotomy, b) damage control surgery, c) hypotensive resuscitation, d) emergency laparotomy, e) possible laparotomy but investigations are required for a diagnosis, f) possible laparotomy but admit for observation and serial examinations, and e) no abdominal injuries warranting a laparotomy.

Resuscitation laparotomy: Resuscitation laparotomy is indicated for the exsanguinating patient in severe shock, not responding to fluid resuscitation with obvious blunt or penetrating abdominal trauma, and no evidence of chest, pelvic or extremity bleeding. The patient requires endotracheal intubation, ventilation and an immediate laparotomy to control the bleeding. The secondary survey could then follow in the ward. Any delay to the operation by attempting to complete limb $\mathrm{x}$-rays, vascular access procedures or other investigations will almost certainly result in early death of the patient. Although resuscitation laparotomies have been performed in the resuscitation room in exceptional circumstances, the procedure should almost always be done in the operating theatre. A resuscitation laparotomy is not indicated if cardiac arrest has occurred $[7,8]$.

Damage Control (DC): Unstable patients should not have extensive and prolonged operations.Once life-saving surgical manoeuvres i.e. stopping ongoing bleeding and contamination have created realistic chance of survival, it is better to stop the operation temporarily with the stated objective of returning the patient to theatre (24-48 hours) later when haemoglobin and circulating volume, hypothermia and clotting deficiencies have improved. This is the basis of damage control surgery where correcting the physiology takes priority over anatomical reconstructive surgery in the exsanguinating critically-ill patient [31]. As survival is determined by physiology rather than anatomy this would avoid or limit the lethal triad of death (hypothermia $<34^{\circ} \mathrm{C}$, acidosis $\mathrm{pH}<7.2$ coagulopathy PT $>16$ s) [31-34]. The survival chance may be lost if attempts at definitive surgery are pursued and a point of no return will be reached where curtailing the operation will be too late and death inevitable. The 'Bad' injury patterns which occur in $10-20 \%$ of trauma victims (high energy, multi-visceral damage, prolonged hypotension, massive blood loss) gets damage control surgery. The scenario is particularly applicable when a patient is bleeding from major liver trauma. Rapidly arresting the haemorrhage by packing the liver is better than attempting definitive repair or resection $[2,7,31]$. Other reasons for performing limited trauma surgery may include; limited surgical expertise, time pressures due to multiple casualties, lack of necessary blood products; inaccessible major venous injury; and other body system injury requiring life-saving surgery $[2-5,8]$.
Hypotensive resuscitation: Hypotensive resuscitation is a different and controversial concept. The aim is to maintain critical organ perfusion of the heart, brain and kidneys, but not disrupt the blood clot that has formed around an injury. Restoring normal blood pressure without first controlling the bleeding by surgical means may actually increase the blood loss and render the situation uncontrollable. Thus, fluid is not given to achieve a 'normal' blood pressure but is either withheld until surgical control is achieved or given to achieve a predetermined 'lower' blood pressure [35]. 'Conscious hypotension' and 'active observation' is utilised while the patient is transferred to the operating theatre for simultaneous bleeding control and resuscitation. Young, fit patients can withstand low blood pressure for surprisingly long periods of time.

Emergency laparotomy: It is practical and good judgement to err on the safe side by urgently arranging for an emergency laparotomy in the operating theatre if any of the following is diagnosed during the clinical examination with or without adjuncts;a) fluid responders but blood in the peritoneal cavity, b) positive FAST in the shocked patient, c) positive DPL, d) peritonism on abdominal examination, e) diaphragmatic rupture, f) protrusion of a viscus through a wound, g) gastrointestinal bleeding, and h) abdominal gunshot wounds [1$3,7,8]$. Many injuries to solid organs can be managed non-operatively. Thus, with the experience and resources of referral trauma centres (a) and (c) may be managed conservatively with 'active observation' [36-38].

Multiple injuries: Prioritisation is important when abdominal injuries occur in association with other injuries. Life-threatening injuries may have to take priority over a minor abdominal injury. If there are other injuries that are overwhelming then it may be inappropriate to embark upon laparotomy. In addition, in a military environment with limited resources prolonged laparotomies should not be undertaken if others with more salvageable injuries would deteriorate in the interim [2,3]. In the multiply injured patient bleeding frequently occurs in more than one body region. Potential bleeding points need to be rapidly identified and their control may require surgery by more than one specialist. The first incision needs to be made to expose and control the most life-threatening bleeding point. This decision needs to be made rapidly which, occasionally, will result in the wrong judgement and rapid correction is equally required $[1-3,7,8]$.

Difficult injury combination: Injuries also occur at the 'junctional' zones between two surgical specialties, e.g. lower ribcage and pelvis or groin and require a careful series of decisions. It is essential that the surgeons discuss the approach in the resuscitation room to prioritise and decide on either sequential or simultaneous surgery. In an austere environment the solitary surgeon has to decide what to do first. For example, the case of a shocked patient with a penetrating wound anterolateral in her lower chest. The chest drain had rapidly drained $700 \mathrm{ml}$ of blood and then stopped. The patient would need an emergency operation and a laparotomy is done first before a thoracotomy.The optimal test to decide whether a laparotomy or thoracotomy is done first is FAST. If FAST shows pericardial effusion and abdominal fluid, a left anterolateral thoracotomy is done first. The thoracotomy can be extended or a second incision for laparotomy made. A second example is a shocked patient with a gunshot wound 
to the groin that have significant bleeding not controlled by direct pressure. The patient needs an emergency operation but through a longitudinal groin incision. If there is difficulty with proximal control a laparotomy is performed and a thigh extension of the incision if difficulty with distal control of haemorrhage [1-3,7,8]. Certain combinations of blunt trauma injuries also require a careful series of decisions. For example, a patient following a car accident responding transiently to intravenous fluids, and investigations reveal free intra-abdominal blood on ultrasound and a pelvic fracture would need an emergency operation. Pelvic fixation to stabilise the pelvic ring disruption and counteract the haemorrhagic factors would be done first before a full midline laparotomy. The second example is a patient who has fallen from a bridge and sustained a disrupted pelvis as well as comminute closed femur fractures, and, did not respond to intravenous fluids. The patient would need an emergency operation but, again external fixation of the pelvis first before femur nailing. A third common example is a shocked patient with free intraabdominal blood and a head injury with fixed and dilated pupil. She would need an emergency operation but a laparotomy first to stop the bleeding and prevent secondary brain injury from hypotension before a craniotomy. The CT scan of the head is done after the laparotomy. Optimally the surgeries can be performed consecutively during the same anaesthetic.

Laparotomy after investigations: It can be very difficult to decide whether a patient needs a laparotomy after trauma, particularly when the patient is haemodynamically stable and has minimal signs of abdominal injury. In some patients, the need for a laparotomy only becomes apparent after investigations. These tests may be particularly useful in patients in whom physical examination is unremarkable (head injury, alcohol, drugs and paralysis, and in patients in whom one is unable to perform serial examinations. Special investigations do not provide absolute answers and the risk of delayed and unnecessary operations will remain. For example, although FAST is an excellent investigation for haemoperitoneum, up to $40 \%$ of patients with an acute haemoperitoneum reveal that bleeding has stopped and that the injuries do not require surgery $[6,28]$. Generally, in a haemodynamically stable patient, if there is any doubt about abdominal injuries or if serial examinations cannot be done or the patient requires transfer, further special and/or organ specific investigations should be done. A CT scan with intravenous contrast is the single most useful investigation because it can assess for intra-peritoneal fluid, solid organ injury, retroperitoneal haematoma, duodenal (with oral contrast) and colorectal injury (with rectal contrast). It is sensitive (92-97.6\%) and specific (98.7\%) [24], and its main advantage is the ability to detect arterial contrast extravasation, uncontained or as a pseudo aneurysm, which predicts the need for surgery or angio-embolisation [39]. Computed tomography also accurately evaluates the retro peritoneum, but it is less sensitive for detecting hollow viscus injuries [30], although detection rates are improving with increasing experience. Computed tomography is also the modality of choice for diagnosing injuries to the diaphragm [40], which may result in major morbidity and mortality if undetected and may not present until many years after the event.A large prospective multicentre study showed that a normal abdominal computed tomography scan has a high negative predictive value $(99.63 \%)$, and it concluded that admission for observation may not be necessary [21]. Free intra-abdominal fluid without solid organ injury is a concern, particularly in neurologically compromised patients, and must be placed in the clinical context with regard to injury patterns and signs of high risk, such as abdominal seat belt marks. In most cases, the fluid is blood and of no further consequence, but occasionally it may be gastrointestinal content from an undetected hollow viscus injury. Such patients should be managed by an experienced surgeon.Intravenous contrast studies assess kidney function and integrity of the urological tract. Oral or rectal watersoluble can similarly be used to assess the integrity of the upper and lower gastrointestinal tracts [24]. Laparoscopy is occasionally used diagnostically to assess for peritoneal breach in penetrating trauma, intraperitoneal blood and hollow viscus content and diaphragmatic lacerations. In carefully selected blunt abdominal trauma patients therapeutic interventions can be safe and feasible [40].

\section{Conclusion}

The understanding and integration of the Advanced Trauma Life Support (ATLS), care of the critically ill surgical patient and definitive surgical trauma skills is particularly important in the management of abdominal trauma. The diagnosis of abdominal injury by clinical examination is unreliable. Although investigation of blunt abdominal trauma is challenging, appropriate and directed investigation will improve diagnosis and allow either early surgical intervention or facilitate conservative management. Where the patient is unstable and an abdominal cause is obvious, immediate surgery is required. Seriously injured patients require an integrated decision-making process.

\section{References}

1. Gilroy D. Deaths from blunt trauma, after arrival at hospital: plus ça change, plus c'est la même chose. Injury. 2005; 36: 47-50.

2. Buckman RF, Scalea TM. International approaches to trauma care. Trauma Quarterly. 1999; 14.

3. Greaves I, Porter KM, Ryan JM. Trauma London: Arnold. 2001.

4. Coupland RM, Parker PJ, Gray RC. Triage of war wounded: the experience of the International Committee of the Red Cross. Injury. 1992; 23: 507-510.

5. National Confidential Enquiry into Perioperative Deaths (NCEPOD). Trauma: who cares? London: NCEPOD. 2007.

6. Janson JO, Yule SR, Loudon MA. Investigation of blunt abdominal trauma. BMJ. 2008; 336: 938-942.

7. American College of Surgeons. ATLS for Doctors: Student Course manual $6^{\text {th }}$ ed. Chicago. 1997.

8. American College of Surgeons. Wilmore DW, et al. Care of the surgical patient. American College of Surgeons/ Scientific American Medicine. New York. 1988.

9. Committee on Trauma. Field Surgery Pocket Book. London: HMSO. 1981.

10. Degiannis E, Boffard K. Duodenal injuries. Brit J Surg. 2000; 87: 1473-1479.

11. Eastham JA, Wilson TG, Ahlering TE. Urological evaluation and management of renal - proximity stab wounds. J Urol. 1003; 150: 177.

12. Miller KS, McAninch JW. Radiographic assessment of renal trauma: our 15year experience. J Urol. 1995; 154: 352-355.

13. Weledji EP, Fokam P, Nzade D, Eyongeta D. Emergency primary repair of grade $\mathrm{V}$ bladder neck injury complicating pelvic fracture. Ann Surglnnov Res. 2014; 8: 4.

14. Ho YH, Pritchett CJ. Blunt abdominal trauma causing a 'degloving injury' to the colon. Injury. 1990; 21: 119-120 
15. Feigenberg Z, Ben-Baruch D, Barak R, Zer M. Penetrating stab wound of the gluteus- a potentially life-threatening injury: case reports. J Trauma. 1992 33: $776-778$.

16. Ferraro FJ, Livingston DH, Odom J, Swan KG, Cormack M, Rush BF. The role of sigmoidoscopy in the management of gunshot wounds to the buttocks. Am Surg. 1993; 59: 350-352.

17. Sinnott R, Rhodes M, Brader A. Open pelvic fractures: an injury for trauma centres. Am J Surg. 1992; 163: 283-287.

18. Doersch KB, Dozier WE. The seat belt syndrome: the seat belt sign, intestinal and mesenteric injuries. Am J Surg. 1968; 116: 831-833.

19. Chichom AM, Weledji EP, Verla VS, Lidwine NM. Diagnostic and therapeutic challenges of isolated small bowel perforations after blunt abdominal injury in low income settings: analysis of twenty three new cases. Injury, Int J Care Injured. 2014; 45: 141-145

20. Myers J. Focused assessment with sonography for trauma (FAST): the truth about ultrasound in blunt trauma. J Trauma. 2007; 62: S28.

21. Livingston DH, Lavery RF, Passannante MR, Skurnick JH, Fabian TC, Fry DE, et al. Admission or observation is not necessary after a negative abdominal computed tomographic scan in patients with suspected blun abdominal trauma: results of a prospective, multiinstitutional trial. J Trauma. 1998; 44: 273-280

22. Isenhour JL, Marx J. Advances in abdominal trauma. Emerg Med Clin North Am. 2007; 25: 713-733.

23. Root HD, Hauser CW, McKinley CR, Lafave GW, Mendiola RP Jr. Diagnostic peritoneal lavage Surgery. 1965; 57: 633-637.

24. Hoff WS, Holevar M, Nagy KK, Patterson L, Young JS, Arrillaga A, et al. Eastern Association for the Surgery of Trauma. Practice management guidelines for the evaluation of blunt abdominal trauma: the EAST practice management guidelines work group. J Trauma. 2002; 53: 602-615.

25. Soyka JM, Martin M, Sloan EP, Himmelman RG, Batesky D, Barret JA. Diagnostic peritoneal lavage: is an isolated WBC count Qeg 500/ mm3predictive of intra-abdominal injury requiring celiotomy in blunt trauma patients? J Trauma. 1990; 30: 874-879.

26. Bain IM, Kirby RM, Tiwari P, Mc Craig J, Cook AL,Oakley PA, et al. Survey of abdominal ultrasound and diagnostic peritoneal lavage for suspected intraabdominal injury following blunt trauma. Injury. 1998; 29: 65-71.

27. Rodriguez C, Barone JE, Wilbanks TO, Rha CK, Miller K. Isolated free fluid on computed tomographic scan in blunt abdominal trauma: a systematic review of incidence and management. J Trauma. 2002; 53: 79-85.
28. Royal College of Radiologists. Making the best use of clinical radiology services: referral guidelines. London: RCR. 2007

29. Shuman WP, Holtzman SR, Bree RL, Bettman MA, Cascani T, Foley WD, et al. American College of Radiology appropriateness criteria. Blunt abdominal trauma. 2005.

30. Bowley DMG, Barker P, Boffard KD. Damage control surgery- concepts and practice. JR Army Med Corps. 2000; 146: 176-182.

31. Cannon WB, Fraser J, Cowell EM. The preventive treatment of wound shock. JAMA. 1918; 70: 618-621.

32. Gubler KD, Gentilello LM, Hassantish SA, Maier RV. The impact of hypothermia on dilutional coagulopathy. J Trauma. 1994; 36: 847-851.

33. Cosgriff N, Moore EE, Sauaia A, Kenny- Moynihan M, Burch JM, Galloway $\mathrm{B}$, et al. Predicting life-threatening coagulopathy in the massively transfused patient. Hypothermia and acidosis revisited. J Trauma. 1997; 42: 857-862.

34. Bickell WH, Wall MJ, Pepe PE, Martin RR, Ginger VF, Allen MK, et al. Immediate versus delayed fluid resuscitation for hypotensive patients with penetrating torso injuries. New England Journal of Medicine. 1994; 331: 1105-1109.

35. Velmahos GC, Toutouzas KG, Radin R, Chan L, Demetriades D. Nonoperative management of blunt injury to solid abdominal organs: a prospective study. Arch Surg. 2003; 138: 844-851.

36. Haan JM, Bocchicchio GV, Kramer N, Scalea TM. Non-operative management of blunt splenic injury: a 5-year experience. J Trauma. 2005; 58: 492-498.

37. Stein DM, Scalea TM. Non- operative management of spleen and liver injuries. J Intensive Care Med. 2006; 21: 296-304.

38. Yao DC, Jeffrey RB, Mirvis SE, Weekes A, Federle MP, Kim C, et al. Using contrast-enhanced helical $\mathrm{CT}$ to visualize arterial extravasation after blunt abdominal trauma: incidence and organ distribution. AJR Am J Roentgenol. 2002; 178: 17-20

39. Brody JM, Leighton DB, Murphy BL, Abbott GF, Vaccaro JP, Jaminas L, et al CT of blunt trauma bowel and mesenteric injury: typical findings and pitfalls in diagnosis. Radio graphics. 2000; 20: 1525-1536.

40. Matsevych OY, Koto MZ, Aldous C. Trauma Laparoscopy: A prospect of skills training (cohort study). Int J Surg. 2018; 55: 117-123.
Austin J Surg - Volume 5 Issue 6 - 2018

ISSN : 2381-9030 | www.austinpublishing group.com

Weledji et al. (C) All rights are reserved
Citation: Weledji EP and Tambe J. Perspectives on the Management of Abdominal Trauma. Austin J Surg. 2018; 5(6): 1147. 